\title{
LETTER
}

CHRONIC MYELOPROLIFERATIVE NEOPLASMS

\section{Direct oral anticoagulants for myeloproliferative neoplasms: results from an international study on $\mathbf{4 4 2}$ patients}

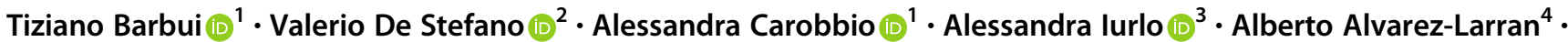 \\ Beatriz Cuevas $^{5}$. Francisca Ferrer Marín $\mathbb{D}^{6}$. Alessandro M. Vannucchi $\mathbb{D}^{7}$. Francesca Palandri $\mathbb{D}^{8}$. \\ Claire Harrison ${ }^{9}$ - Hassan Sibai ${ }^{10}$ - Martin Griesshammer ${ }^{11}$. Massimiliano Bonifacio ${ }^{12}$ • Elena M. Elli ${ }^{13}$. \\ Chiara Trotti $^{14}$ - Steffen Koschmieder $\mathbb{I}^{15}$. Giuseppe Carli ${ }^{16} \cdot$ Giulia Benevolo $^{17}$ - Jean-Christophe lanotto $\mathbb{D}^{18}$. \\ Swati Goel ${ }^{19}$ - Anna Falanga ${ }^{20,21}$ - Silvia Betti ${ }^{2}$ - Daniele Cattaneo ${ }^{3}$ - Eduardo Arellano-Rodrigo ${ }^{4}$ - Lara Mannelli ${ }^{7}$. \\ Nicola Vianelli ${ }^{8} \cdot$ Andrew Doyle $\mathbb{D}^{9} \cdot$ Vikas Gupta $^{10} \cdot$ Kai Wille $\mathbb{D}^{11} \cdot$ Douglas Tremblay $^{22} \cdot$ John Mascarenhas $\mathbb{D}^{22}$
}

Received: 17 February 2021 / Revised: 14 April 2021 / Accepted: 29 April 2021 / Published online: 19 May 2021

(c) The Author(s), under exclusive licence to Springer Nature Limited 2021

\section{To the Editor:}

In the Philadelphia-negative myeloproliferative neoplasms (MPN), the major burden of disease is the elevated risk of thrombosis [1]. Moreover, MPN patients are concomitantly prone to bleeding [1], making antithrombotic therapy challenging. Regarding atrial fibrillation (AF), very scarce

These authors contributed equally: Tiziano Barbui, Valerio De Stefano

Supplementary information The online version contains supplementary material available at https://doi.org/10.1038/s41375021-01279-1.

Tiziano Barbui

tbarbui@fondazionefrom.it

FROM Research Foundation, ASST Papa Giovanni XXIII, Bergamo, Italy

2 Section of Hematology, Department of Radiological and Hematological Sciences, Catholic University, Policlinico Universitario "A. Gemelli” IRCCS, Roma, Italy

3 Fondazione IRCCS Ca' Granda Ospedale Maggiore Policlinico, Milano, Italy

4 Hospital Clinic, Barcelona - GEMFIN, Barcelona, Spain

5 Hospital de Burgos - GEMFIN, Burgos, Spain

6 Hospital Morales Messeguer, Murcia - GEMFIN, Murcia, Spain

7 CRIMM, Center Research and Innovation of Myeloproliferative Neoplasms, University of Florence, AOU Careggi, Firenze, Italy

8 Institute of Hematology "L. \& A. Seragnoli”, IRCCS Azienda Ospedaliero-Universitaria di Bologna, Bologna, Italy

9 Guy's and St Thomas' NHS Foundation Trust, London, UK

10 Princess Margaret Cancer Centre, Toronto, ON, Canada

11 Johannes Wesling Medical Center, University of Bochum, data are available about treatment outcomes with vitamin-K antagonists (VKAs) or direct oral anticoagulants (DOACs) $[2,3]$. On the other hand, VKAs partially prevent recurrent venous thromboembolism (VTE), and the incidence of either recurrent thrombosis and major bleeding is still unacceptable high [4].

DOACs emerged as a treatment of choice for secondary prevention of VTE and thrombosis prophylaxis in AF in the general population, and may be an attractive alternative to VKAs in MPN. However, evidence of their efficacy and safety in MPN is very limited and based on few retrospective observational studies [3, 5-7], and on scattered information from controlled trials of DOACs versus VKAs.

Bochum, Germany

12 Department of Medicine, Section of Hematology, University of Verona, Verona, Italy

13 Hematology Division and Bone Marrow Unit, San Gerardo Hospital, ASST Monza, Monza, Italy

14 Dipartimento di Medicina Molecolare, Università degli Studi di Pavia, Pavia, Italy

15 Faculty of Medicine, Department of Hematology, Oncology, Hemostaseology, and Stem Cell Transplantation, RWTH Aachen University, Aachen, Germany

16 Ospedale San Bortolo, Vicenza, Italy

17 Hematology, AOU Città della Salute e della Scienza di Torino, Turin, Italy

18 Service d'Hématologie Clinique, Institut de Cancéro-Hématologie, CHRU de Brest, Brest, France

19 Albert Einstein Montefiore Medical Center, New York, NY, USA

20 ASST Papa Giovanni XXIII, Bergamo, Italy

21 School of Medicine, University of Milan Bicocca, Monza, Italy

22 Icahn School of Medicine at Mount Sinai, New York, NY, USA 
On the other hand, randomized clinical trials comparing DOACs to VKAs are hardly feasible in MPN, given their rarity and low interest by the pharmaceutical companies. Nonetheless, DOACs are increasingly being used in the clinical practice of patients with MPN. Therefore, we considered useful to verify whether there were indications of suspected excesses of risk or decreased efficacy in the realworld practice in MPN.

We conducted an observational, multicenter, international study in 442 patients to estimate the incidence and risk factors for thrombotic and bleeding complications in MPN patients with AF and VTE treated with a DOAC in real-world clinical practice. The MPN-DOACs study (ClinicalTrials.gov: NCT04192916) is a European LeukemiaNet project conducted across 19 centers (16 European, 2 US, and 1 Canadian). Eligible patients were consecutive adults (aged $\geq 18$ years) with MPN recruited in the prepandemic SARS-CoV-2 infection and diagnosed from January 1, 2005 to December 31, 2018 treated with any DOAC (i.e., dabigatran, apixaban, rivaroxaban, edoxaban) for (i) a concomitant diagnosis of AF or (ii) the occurrence of a VTE. The decision concerning indication for and duration of anticoagulant treatment with DOACs had been independently established by the attending physician, given the observational nature of the study. The primary outcome was the occurence of any of the following: an arterial thrombotic event, a venous thrombotic event or a major bleeding event, as previously reported [4]. We included MPN patients who were treated with a DOAC, either for primary or secondary prophylaxis against systemic embolism, in the case of AF, or for prevention of recurrent VTE. Written informed consent of participants was collected whenever possible according to each Country's regulation. We calculated the annual incident rate (IR) of first or recurrent thrombosis, bleeding or death, and the cumulative probability as a function of time using the Kaplan-Meier method, comparing groups with the log-rank test. The univariate analysis compared patients with or without thrombosis or bleeding for meaningful covariates and then selected the ones for multivariate analysis that showed a difference with a $p$ value $<0.05$.

Characteristics of MPN patients with AF and VTE at the start of treatment with a DOAC are provided in Table S1. Compared to VTE patients, patients with AF were older $(15.8 \%>80$ years), and were enriched in ET (91/203, $44.8 \%$ ). At least one cardiovascular risk factor was present in $70 \%$ of cases, particularly in $\mathrm{AF}(176 / 203,86.7 \%)$ compared with VTE patients $(133 / 235,56.6 \%)(p<0.001)$. No significant difference in the driver mutation status between the two groups was documented. History of bleeding was present in 35 of 442 patients (7.9\%) and was reported in the same proportion of ET, PV, and MF, and similarly in AF and VTE groups.
DOACs were prescribed in 203 patients with $\mathrm{AF}$ (45.9\%) and 239 patients with VTE $(54.1 \%)$ after a median interval of 4.4 years (IQR: 0.4-9.6) since MPN diagnosis. DOAC treatment details and the concomitant other drugs are reported in Table S2. Cytoreduction was prescribed in 398 patients $(90 \%)$, hydroxyurea and ruxolitinib being the most frequent. In 60 patients (13.6\%), DOACs were discontinued after a median duration of 1.1 years (IQR: 0.4-2.2). Reason for discontinuation is presented in Table S3.

In AF patients, after a follow-up of 1.7 years (IQR: $0.8-3.1)$, ten major thrombotic events were reported $(2.1 \%$ patients-year [pts-yr]), of which six occurred in 166 patients (3.6\%) with no history of previous thrombosis accounting for an annual IR of $1.5 \%$ (Table 1). Five of these events were arterial (TIA, MI) and one DVT. In 4 of 37 patients (10.8\%) who had a prior history of stroke/TIA $(24 / 37,65 \%)$ and other arterial or venous episodes, the annual IR was remarkably higher, accounting for $4.6 \%$ and significantly associated with previous arterial thrombosis $(p=0.026$, Table S4). Figure 1A illustrates the different cumulative incidence of thrombosis in this latter group, which reached a plateau after around 15 months of observation. No ischemic stroke was recorded; the IR of TIA was $0.6 \%$ pts-yr in the patients without previous thrombosis and $1.1 \%$ pts-yr in those with previous thrombosis. Thus, the IR of ischemic cerebrovascular events (ICVE), although derived from a limited number of patients, compares favorably with the IR of systemic embolism reported in non-MPN patients with $\mathrm{AF}$, where the IR of ICVE during primary prophylaxis with VKAs or DOACs is $1.2-1.8 \%$ and $1.0-1.4 \%$ pts-yr, respectively [8-11]. The IR of systemic embolism using VKAs or DOACs after an ICVE is $2.7-3.2 \%$ pts-yr and $2.0-2.8 \%$ pts-yr, respectively [8-11]. However, the efficacy in preventing ICVE can be due to the concomitant use of hydroxyurea ( $82 \%$, Table S2), which has been reported to be an independent protective factor for recurrence in MPN patients with previous ICVE [12].

During the same observation period, among 239 patients with VTE, 22 recurrent events (9.2\%) were registered, of which 17 occurred in venous and 5 in arterial districts, for an IR of 4.5\% pts-yr (Table 1). Among 158 patients with venous thrombosis of the lower extremity, 16 recurrences (annual IR 5.1\%) occurred predominantly in the venous districts, independently on whether they occurred formerly in lower extremity DVT (5.1\% pts-yr) or splanchnic vein thrombosis (3.2\% pts-yr). Interestingly, this recurrence rate is comparable to that found in our series of 206 MPN patients with VTE and 181 MPN patients with SVT receiving VKAs (IR 5.3\% and 3.9\% pts-yr, respectively) $[4,13]$. Figure $1 \mathrm{~A}$ illustrates the cumulative incidence of events over time. In univariate analysis of all VTE episodes (Table S4), no impact was seen on recurrences by the initial site of thrombosis $(p=0.76)$, type of DOAC $(p=0.55)$, 
Table 1 Incidence of thrombosis and major bleeding after DOAC initiation.

\begin{tabular}{|c|c|c|c|c|}
\hline Indication & $\begin{array}{l}\text { Thrombosis after DOAC start, } \mathrm{n} / \mathrm{N} \\
\text { (\%) [type] }\end{array}$ & $\begin{array}{l}\text { Incidence rate } \% \\
\text { pt-yrs }(95 \% \mathrm{CI})\end{array}$ & $\begin{array}{l}\text { Bleeding after DOAC } \\
\text { start, } \mathrm{n} / \mathrm{N}(\%) \text { [type] }\end{array}$ & $\begin{array}{l}\text { Incidence rate \% } \\
\text { pt-yrs }(95 \% \mathrm{CI})\end{array}$ \\
\hline Atrial fibrillation, $N=203$ & $\begin{array}{l}10(\mathbf{4 . 9 \%}) \\
\text { Arterial } n=7(3.4 \%) \\
\text { Venous } n=3(1.5 \%)\end{array}$ & $\begin{array}{l}2.1(\mathbf{1 . 1}-\mathbf{3 . 9}) \\
1.5(1.7-3.0) \\
0.6(0.2-1.9)\end{array}$ & $14(6.9 \%)$ & $3.0(1.8-5.0)$ \\
\hline $\begin{array}{l}\text { - No previous thrombosis, } \\
\quad N=166\end{array}$ & $\begin{array}{l}6(3.6 \%) \\
{[3 \text { TIA, } 2 \text { AMI, } 1 \text { DVT] }}\end{array}$ & $1.5(0.7-3.4)$ & $\begin{array}{l}14(8.4 \%) \text { [6 GI, } 5 \\
\text { Muscle, } 3 \text { other] }\end{array}$ & $3.0(1.8-5.0)$ \\
\hline - Previous thrombosis, $N=37$ & $4(10.8 \%)$ [1 TIA, 1 AMI, 2 DVT] & $4.6(1.7-12.2)$ & $0(0.0 \%)$ & $0(0.0-0.0)$ \\
\hline - Stroke/TIA, $N=24$ & $2(8.3 \%)$ [1 TIA, 1 DVT] & & - & - \\
\hline $\begin{array}{l}\text { - Other art thrombosis, } N=9 \\
\text { (8 AMI; } 1 \text { PAT) }\end{array}$ & $2(22.2 \%)$ [1 AMI, 1 DVT] & & - & - \\
\hline $\begin{array}{l}\text { - Venous thrombosis, } N=4 \\
\text { (3 DVT }+/- \text { PE, } 1 \text { SVT) }\end{array}$ & $0(0.0 \%)$ & & - & - \\
\hline $\begin{array}{l}\text { Venous thromboembolism, } \\
N=\mathbf{2 3 9}\end{array}$ & $\begin{array}{l}22(\mathbf{9 . 2 \%}) \\
\text { Arterial } n=5(2.1 \%) \\
\text { Venous } n=17(7.1 \%)\end{array}$ & $\begin{array}{l}4.5(\mathbf{2 . 9}-6.8) \\
1.0(0.4-2.3) \\
3.4(2.1-5.5)\end{array}$ & $12(5.0 \%)$ & $2.3(1.3-4.1)$ \\
\hline $\begin{array}{l}- \text { DVT of the lower extremitys } \\
\quad+/-\mathrm{PE}, n=158\end{array}$ & $\begin{array}{l}16(10.1 \%)[10 \mathrm{DVT}+/-\mathrm{PE}, 1 \text { portal } \\
\text { vein, } 1 \text { cerebral vein, } 1 \text { AMI, } 1 \text { stroke, } \\
1 \text { other }]\end{array}$ & $5.1(3.1-8.3)$ & $\begin{array}{l}9(5.7 \%) \text { [5 GI, } 2 \\
\text { muscle, } 1 \text { CNS, } 1 \text { other] }\end{array}$ & $2.7(1.4-5.2)$ \\
\hline $\begin{array}{l}\text { - Splanchnic vein thrombosis, } \\
n=51\end{array}$ & $\begin{array}{l}4(7.8 \%)[1 \text { portal vein, } 1 \text { TIA, } 1 \text { PAT, } \\
1 \text { mesenteric artery] }\end{array}$ & $3.2(1.2-8.6)$ & $1(2.0 \%)[\mathrm{GI}]$ & $0.8(0.1-5.5)$ \\
\hline $\begin{array}{l}\text { - Cerebral vein thrombosis, } \\
\quad n=13\end{array}$ & $1(7.7 \%)$ [1 mesenteric vein] & & $0(0.0 \%)$ & - \\
\hline $\begin{array}{l}\text { - Other vein thrombosis, } \\
\quad n=17^{\mathrm{a}}\end{array}$ & $1(5.9 \%)$ [1 cerebral vein] & & $\begin{array}{l}2(11.8 \%)[2 \\
\text { Menorrhagia] }\end{array}$ & $6.5(1.6-26.2)$ \\
\hline
\end{tabular}

AMI acute myocardial infarction, TIA transient ischemic attack, $P A T$ peripheral arterial thrombosis, art arterial, $D V T$ deep vein thrombosis, $P E$ pulmonary embolism, GI gastrointestinal, $C N S$ central nervous system.

${ }^{a}$ Upper extremity DVT, $N=10$; superficial vein thrombosis, $N=7$.

We highlighted in bold the first row of either AF and VTE patients to underline the overall estimates of the categories (all other rows present estimates of subgroups).

and MPN phenotype $(p=0.67)$. On the contrary, significant risk factors were previous arterial thrombosis $(p=0.047)$, hypertension $(p=0.017)$, and diabetes $(p=0.035)$. However, none of these risk factors retained significance in multivariate analysis after correction for age and sex.

Almost all patients were receiving cytoreductive drugs (Table S2) so that we were unable to confirm the conclusion of a recent meta-analysis [14] that the risk of recurrence after VTE in MPN was lowest for patients receiving oral anticoagulation plus cytoreduction. Therefore, studies in this setting are urgently needed considering that the IR of recurrent VTE in the present series of MPN patients receiving a DOAC is twice as high as in non-MPN patients treated with DOACs for more than 3 months after the acute VTE event (3.4\% versus $1.5 \%$ pts-yr, respectively) [15].

In total, 26 major hemorrhagic events (14 in AF and 12 in VTE) were diagnosed, primarily in the gastrontestinal tract, accounting for an annual rate of $3 \%$ and $2.3 \%$ in $\mathrm{AF}$ and VTE, respectively (Table 1). In univariate analysis, the proportion of MF patients who bled was significantly higher than the other MPN phenotypes (Table S5). Among the four DOACs, dabigatran was more frequently associated with bleeding than the other three $(7 / 26,27 \%$ vs. $43 / 416,10 \%$, $p=0.010)$. Notably, patients who bled were more frequently affected by MF $(p=0.005)$. In a multivariate analysis that included the correction for age, sex, AF, or VTE indication, both $\mathrm{MF}$ diagnosis $(\mathrm{HR}=3.6,95 \% \mathrm{CI}: 1.6-8.2$, $p=0.002)$ and dabigatran use $(\mathrm{HR}=3.8,95 \% \mathrm{CI}: 1.5-9.7$, $p=0.005)$ retained statistical significance. Figure $1 \mathrm{~B}, \mathrm{C}$ illustrates the cumulative incidence of these events during the observation.

Overall, 39 deaths were registered $(8.8 \%)$ for an IR of $3.8 \%$ pts-yr (95\% CI: 2.8-5.3), with no difference between patients with AF or VTE $(p=0.98)$. Causes of death are reported in Table S6.

In conclusion, we acknowledge the intrinsic limitations of our investigation, due to its retrospective design, and a selection bias of patients treated with DOACs cannot be excluded. However, the strength is the relatively large number of patients in a rare condition and its generalizability, given that these patients were followed-up in multiple international healthcare settings. Moreover, the distribution of patients receiving these drugs was wellbalanced among the MPN phenotypes allowing a 
Fig. 1 Cumulative incidence of thrombosis and bleeding.

Kaplan-Meier cumulative incidence of (A) thrombosis by indication for the treatment with DOAC and previous thrombosis; (B) bleeding by MPN phenotype and (C) bleeding by DOAC drug. $p$ values calculated by the log-rank test.

A

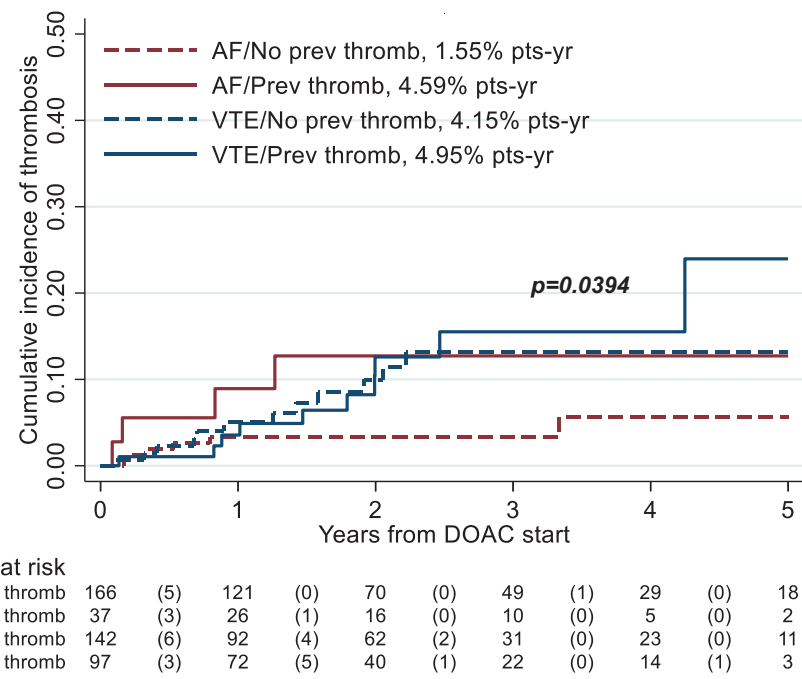

B

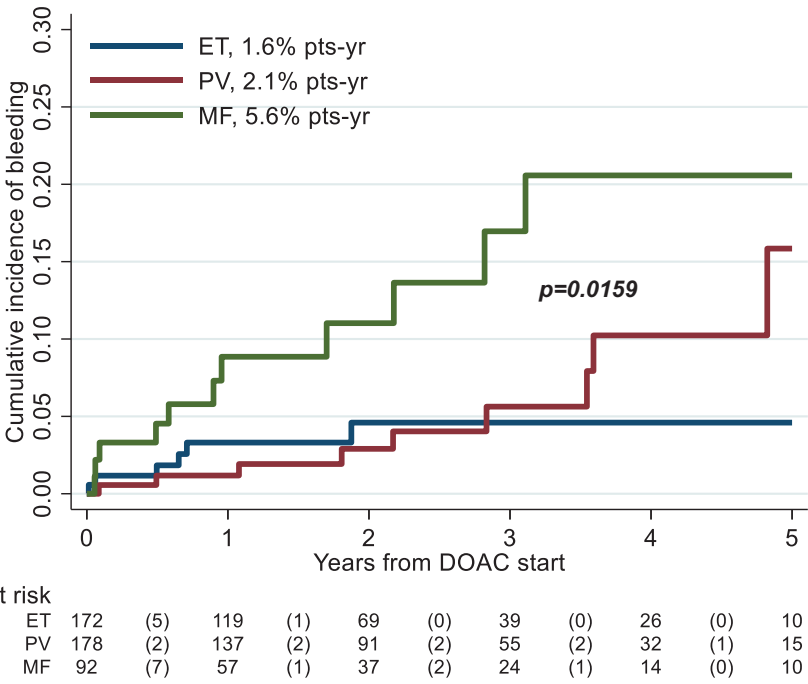

C

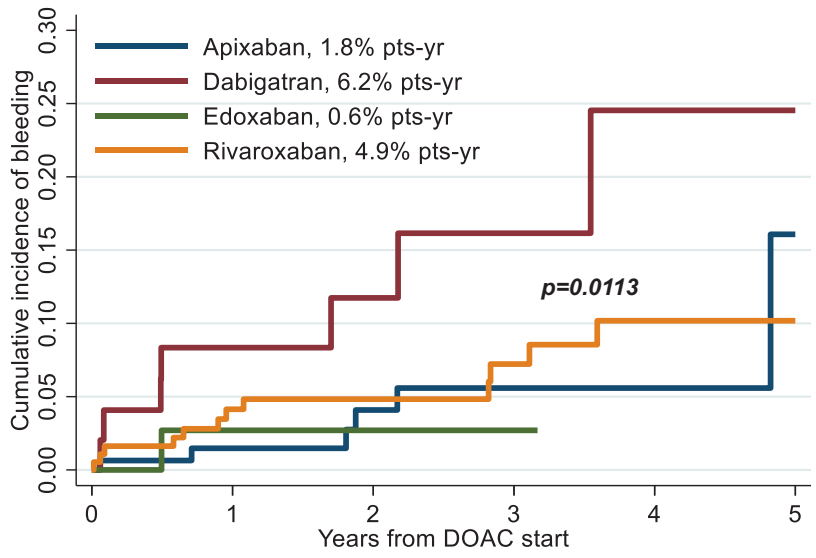

Number at risk

Apixaban 157

$\begin{array}{rccccccccccc}\text { Apixaban } & 157 & (2) & 110 & (2) & 69 & (1) & 32 & (0) & 17 & (1) & 6 \\ \text { Dabigatran } & 50 & (4) & 38 & (1) & 21 & (1) & 12 & (1) & 8 & (0) & 5 \\ \text { Edoxaban } & 48 & (1) & 24 & (0) & 6 & (0) & 1 & (0) & 0 & (0) & 0 \\ \text { Rivaroxaban } & 187 & (7) & 141 & (1) & 101 & (2) & 73 & (2) & 47 & (0) & 24\end{array}$

comparison of DOACs efficacy and risk. Overall, DOACs and VKAs seem to have a substantially similar risk-benefit profile for the prophylaxis of VTE in MPN. The favorable efficacy in preventing ICVE in AF may be confounded by the use of hydroxyurea. We highlight the significant bleeding tendency in PMF and in association with 
dabigatran. Thus, DOACs could represent a possible alternative to VKAs for antithrombotic prophylaxis given the advantage in ease of administration and improved patient convenience.

Acknowledgements MPN-DOACs is sponsored by FROM-Fondazione per la Ricerca Ospedale di Bergamo and endorsed by the AIRC - Gruppo Italiano Malattie Mieloproliferative (AGIMM) with the program number \#21267, progetto Mynerva.

Author contributions TB designed the study, interpreted the data, and wrote the paper. VDS. interpreted the data and wrote the paper. AC accessed and verified the data and performed the statistical analysis. JM and AMV critically revised the paper. AI, AA-L, BC, FFM, AMV, FP, $\mathrm{CH}, \mathrm{HS}, \mathrm{MG}, \mathrm{MB}$, EME, CT, SK, GC, GB, J-CI, SG, AF, SB, DC, EAR, LM, NV, AD, VG, KW, DT collected patient's data of the study. All authors discussed the results and implications and commented on the manuscript, giving the final approval of the version to be published.

\section{Compliance with ethical standards}

Conflict of interest The authors declare no competing interests.

Publisher's note Springer Nature remains neutral with regard to jurisdictional claims in published maps and institutional affiliations.

\section{References}

1. Rungjirajittranon $\mathrm{T}$, Owattanapanich $\mathrm{W}$, Ungprasert $\mathrm{P}$, Siritanaratkul N, Ruchutrakool T. A systematic review and meta-analysis of the prevalence of thrombosis and bleeding at diagnosis of Philadelphianegative myeloproliferative neoplasms. BMC Cancer. 2019;19:184.

2. de Freitas AS, Alvarez-Larrán A. Risk of thrombosis and hemorrhage in patients with polycythemia vera and atrial fibrillation treated with prophylactic oral anticoagulants. Ann Hematol. 2016;95:1903-4.

3. Serrao A, Breccia M, Napolitano M, Fiori L, Santoro M, Scalzulli E, et al. A multicenter real-life study on anticoagulant treatment with direct oral anticoagulants in patients with $\mathrm{Ph}$ negative myeloproliferative neoplasms. Am J Hematol. 2020;5:E329-32.

4. De Stefano V, Ruggeri M, Cervantes F, Alvarez-Larrán A, Iurlo A, Randi ML, et al. High rate of recurrent venous thromboembolism in patients with myeloproliferative neoplasms and effect of prophylaxis with vitamin K antagonists. Leukemia. 2016;30:2032-8.
5. Curto-Garcia N, Doyle AJ, Breen KA, McLornan DP, Radia DH, Hunt BJ, et al. Outcomes of patients receiving direct oral anticoagulants for myeloproliferative neoplasm-associated venous thromboembolism in a large tertiary centre in the UK. Br J Haematol. 2020;189:e79-81.

6. Tremblay D, Vogel AS, Moshier E, Hoffman R, Kremyanskaya $\mathrm{M}$, Zhou S, et al. Outcomes of splanchnic vein thrombosis in patients with myeloproliferative neoplasms in a single center experience. Eur J Haematol. 2020;104:72-3.

7. Huenerbein K, Sadjadian P, Becker T, Kolatzki V, Deventer E, Engelhardt C, et al. Direct oral anticoagulants (DOAC) for prevention of recurrent arterial or venous thromboembolic events (ATE/VTE) in myeloproliferative neoplasms. Ann Hematol. 2020. https://doi.org/10.1007/s00277-020-04350-6.

8. Connolly SJ, Ezekowitz MD, Yusuf S, Eikelboom J, Oldgren J, Parekh A, et al. Dabigatran versus warfarin in patients with atrial fibrillation. N Engl J Med. 2009;361:1139-51.

9. Granger CB, Alexander JH, McMurray JJ, Lopes RD, Hylek EM, Hanna M, et al. Apixaban versus warfarin in patients with atrial fibrillation. N Engl J Med. 2011;365:981-92.

10. Hankey GJ, Patel MR, Stevens SR, Becker RC, Breithardt G, Carolei A, et al. Rivaroxaban compared with warfarin in patients with atrial fibrillation and previous stroke or transient ischaemic attack: a subgroup analysis of ROCKET AF. Lancet Neurol. 2012;11:315-22.

11. Giugliano RP, Ruff CT, Braunwald E, Murphy SA, Wiviott SD, Halperin JL, et al. Edoxaban versus warfarin in patients with atrial fibrillation. N Engl J Med. 2013;369:2093-104.

12. De Stefano V, Carobbio A, Di Lazzaro V, Guglielmelli P, Iurlo A, Finazzi MC, et al. Benefit-risk profile of cytoreductive drugs along with antiplatelet and antithrombotic therapy after transient ischemic attack or ischemic stroke in myeloproliferative neoplasms. Blood Cancer J. 2018;8:25.

13. De Stefano V, Vannucchi AM, Ruggeri M, Cervantes F, AlvarezLarrán A, Iurlo A, et al. Splanchnic vein thrombosis in myeloproliferative neoplasms: risk factors for recurrences in a cohort of 181 patients. Blood Cancer J. 2016;6:e493.

14. Hamulyàk EN, Daams JG, Leebeek FWG, Biemond BJ, Te Boekhorst PAW, Middeldorp S, et al. A systematic review of antithrombotic treatment of venous thromboembolism in patients with myeloproliferative neoplasms. Blood Adv. 2021;5: 113-21.

15. Wu C, Alotaibi GS, Alsaleh K, Linkins LA, McMurtry MS. Casefatality of recurrent venous thromboembolism and major bleeding associated with aspirin, warfarin, and direct oral anticoagulants for secondary prevention. Thromb Res. 2015;135:243-8. 